\title{
Estimation of natural frequencies of the laboratory truss structure with output only subspace and frequency-domain techniques
}

\author{
Ján Kortiš ${ }^{1, *}$, Lukáš Maliar ${ }^{2}$ and Luboš Daniel ${ }^{1}$ \\ ${ }^{1}$ University of Žilina, Reserach Center of University of Žilina, Univerzitná 8215/1, 01026 Žilina, \\ Slovak Republic \\ ${ }^{2}$ University of Žilina, Department of Structural Mechanics and Applied Mathematics, Univerzitná \\ 8215/1, 01026 Žilina, Slovak Republic
}

\begin{abstract}
Non-destructive methods of structural testing are preferred to identify their current state because they do not damage the structure. Modal analysis is one of these methods that can be used for a comprehensive assessment of structural measurements. Its main advantage is that it can be used for variety applications. The paper presents an application of operational modal analysis to determine modal parameters of a laboratory steel truss structure. Its goal is to make contribution to the still unfinished development of modal analysis in the area of comprehensive application. There is important to try to use it and especially try to derive some basic generally applicable rules. It is an important step for experimental modal analysis before it can be widely used and accepted in engineering applications.
\end{abstract}

\section{Introduction}

When applying classical techniques of experimental operational analysis (EMA) to large constructions such as bridges and buildings, we encounter the problem of choosing an efficient excitation source [1]. In most cases, the requirements for excitation sources are so great that it is not technically feasible, or the cost of the excitation device is extreme [2]. For this reason, operational modal analysis (OMA) techniques are used [3]. In this case, the source of excitation is either ambient vibrations such as wind and traffic, or directly the operating excitation. The disadvantage of using such a procedure is that the excitation is assumed to have a character of random noise, otherwise the measurement is influenced by the excitation character itself and this assumption is not fully satisfied. This fact is important to take into account during the analysis. Two classic modern approaches are presented and compared in the paper: Frequency Domain Decomposition (FDD) $[4,5]$ and generalized multi-setup Stochastic Subspace Identification method (SSI) [6]. The results of measurement and identified natural frequencies are clearly compared in the table. For the FDD method, also identified mode shapes are presented.

\footnotetext{
*Corresponding author: jan.kortis@uniza.sk
} 


\title{
2 Theoretical background
}

\author{
Frequency Domain Decomposition (FDD)
}

The frequency domain decomposition is an output-only system identification technique popular in civil engineering, in particular in structural health monitoring. As an output-only algorithm, it is useful when the input data is unknown. FDD is a modal analysis technique which generates a system realization using the frequency response given multi-output data. In FDD identification, the first step is to estimate the PSD matrix at discrete frequencies $\omega=\omega_{i}$. This matrix is then decomposed by taking the SVD of the matrix

$$
\widehat{\mathbf{G}_{l}}=\mathbf{U}_{i} \mathbf{S}_{i} \mathbf{U}_{i}{ }^{H}
$$

where the matrix $\mathbf{U}_{\mathrm{i}}$ is a unitary matrix holding the singular vectors $\mathbf{u}_{\mathrm{ij}}$, and $\mathbf{S}_{\mathrm{i}}$ is a diagonal matrix holding the scalar singular values $s_{i j}$. A peak corresponding to the $k$ th mode in the spectrum dominates. Thus, the corresponding singular vector $\mathbf{u}_{i 1}$ is an estimate of the mode shape

$$
\widehat{\Psi}=\mathbf{u}_{i 1}
$$

If two modes are dominating, the first singular vector will be a good estimate of the strongest mode. In the case where two modes are orthogonal, two singular vectors are unbiased estimates of the corresponding mode shape vectors. Two modes that are not orthogonal leads to small bias on the mode shape estimate of the dominant mode, but the strong bias on the mode shape estimate of the weak mode [4]. It can help to estimate two close modes at two different frequency lines.

\section{Subspace Identification Method (SSI-COV)}

Stochastic subspace-based system identification methods are tools for the identification of linear time-invariant systems, fitting a linear model to input/output or output-only measurements taken from a system [7]. The excitation of the system is assumed to be noise with certain properties. These methods can be divided according to the type of data that they require. The SSI-COV method identifies a stochastic state-space model from crosscorrelations of output-only data. Consider linear multivariable time invariant systems described by a discrete time state space model

$$
\begin{aligned}
\mathbf{x}_{k+1} & =\mathbf{A} \mathbf{x}_{k}+\mathbf{w}_{k} \\
\mathbf{y}_{k} & =\mathbf{C} \mathbf{x}_{k}+\mathbf{v}_{k}
\end{aligned}
$$

where the matrices $\mathbf{A}$ and $\mathbf{C}$ are the state transition and observation matrices, respectively, and $w_{k}$ and $v_{k}$ are unobserved output disturbances [8]. These are assumed to be zero mean Gaussian white noise. Denote a matrix $\mathcal{H}$ as subspace matrix, whose estimate $\widehat{\mathcal{H}}$ is built from the cross-correlations between output data of the system (3). The subspace matrix enjoys the factorization property

$$
\mathcal{H}=\mathbf{W O Z}
$$


into the matrix of observability

$$
\mathcal{O} \stackrel{\text { def }}{=}\left[\begin{array}{c}
\mathbf{C} \\
\mathbf{C A} \\
\mathbf{C A}^{2} \\
\vdots \\
\mathbf{C A}^{p}
\end{array}\right]
$$

Let the matrix $\mathbf{G}$ represent cross-correlation between the state and the outputs. In factorization property (4), the matrix $\mathbf{W}$ is the identity matrix and

$$
Z=\mathcal{C} \stackrel{\text { def }}{=}\left[\begin{array}{lllll}
\mathbf{G} & \mathbf{A G} & \mathbf{A}^{2} \mathbf{G} & \ldots & \mathbf{A}^{\mathrm{q}-1} \mathbf{G}
\end{array}\right]
$$

is the well-known controllability matrix. The observation matrix $\mathbf{C}$ is then found in the first block-row of the observability matrix $\mathcal{O}$. The state transition matrix $\mathbf{A}$ is obtained from the shift invariance property of $\mathcal{O}$, namely as the least squares solution of

$$
\mathcal{O}^{\uparrow} \mathbf{A}=\mathcal{O}^{\downarrow} \text {, where } \mathcal{O}^{\uparrow} \stackrel{\text { def }}{=}\left[\begin{array}{c}
\mathbf{C} \\
\mathbf{C A} \\
\vdots \\
\mathbf{C A}^{p-1}
\end{array}\right], \mathcal{O}^{\downarrow} \stackrel{\text { def }}{=}\left[\begin{array}{c}
\mathbf{C A} \\
\mathbf{C A}^{2} \\
\vdots \\
\mathbf{C A}^{p}
\end{array}\right]
$$

These two matrices $\mathbf{A}$ and $\mathbf{C}$ are used to compute modal parameters.

$$
\operatorname{det}(\mathbf{A}-\lambda \mathbf{I})=0, \quad \mathbf{A} \phi_{\lambda}=\lambda \phi_{\lambda}, \boldsymbol{\Psi}=\mathbf{C} \phi_{\lambda}
$$

\section{Experimental measurements}

The design of the laboratory steel truss structure used to compare the two approaches to the measurement signal evaluation is described in detail in [9]. Sixteen Brüel \& Kjær (BK) type 4508-B-002 accelerometers were used to measure the dynamic response of the structure to the random excitation. Four of them were placed as reference sensors and the rest was moved so that responses were measured in all 84 degrees of freedom. The special technique described in [6] was used to join the individual measurements for SSI-COV method and the non-parametric assembly approach described in [10] was used for FDD method. The position of sensors of the individual measurement sets is shown in the Fig. 1.

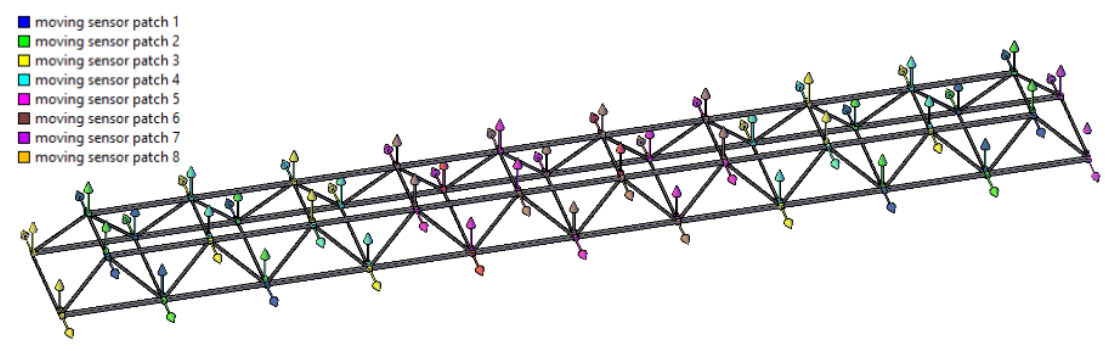

Fig. 1. Model of the steel truss structure with measured points. 
The structure was excited by manual impulses at randomly chosen positions of the steel truss structure. The measurement in each setup lasted 300 seconds, and the sampling rate for each time record was selected as $2048 \mathrm{~Hz}$. The measurement results are processed using two methods to identify the natural frequencies described in the previous section. Mode shapes have also been identified and presented in the article.

\section{Comparison of experimental measurements}

The measured data are preprocessed by lowpass filter with an uppercut of frequency 256 $\mathrm{Hz}$. The decimating factor is selected with a value of 4 which means that the desired frequency range after decimation is from 0 to $256 \mathrm{~Hz}$. The resolution of the spectral plot is $4096 \mathrm{~Hz}$. The same preprocessing setting is used for both used methods. It is possible to estimate modes in the frequency range 0 to $110 \mathrm{~Hz}$, but only the first eight modes are presented.

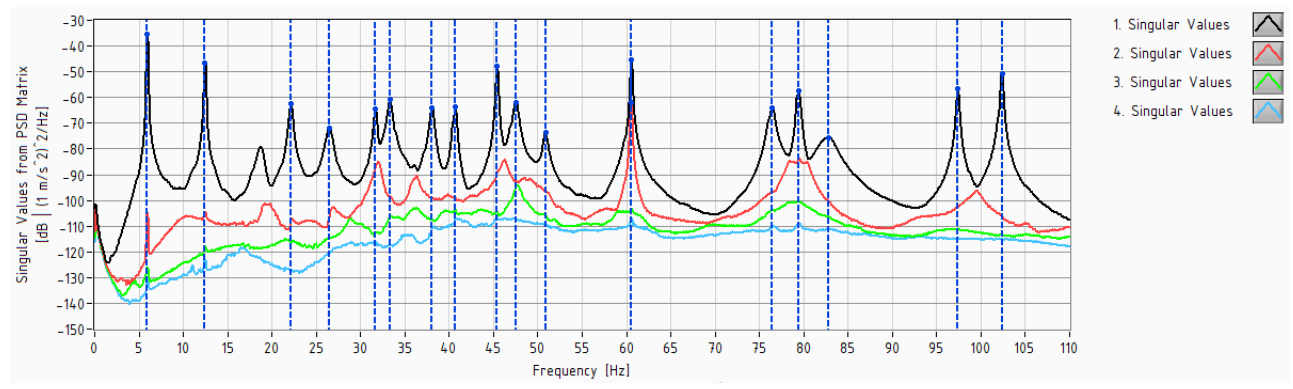

Fig. 2. The results of FDD method.

The singular value functions obtained by processing the measured data using the FDD method are presented in Fig. 2. Functions describing the waveform of the singular values that are used to determine the natural frequencies. All modes are possible to estimate from the first singular line except the two closely spaced modes near $60 \mathrm{~Hz}$, which must be estimated from the first two singular lines. From FE-model it was determined the third peak near $18 \mathrm{~Hz}$ belongs to the longitudinal mode shape, thus it is not chosen for analysis task.

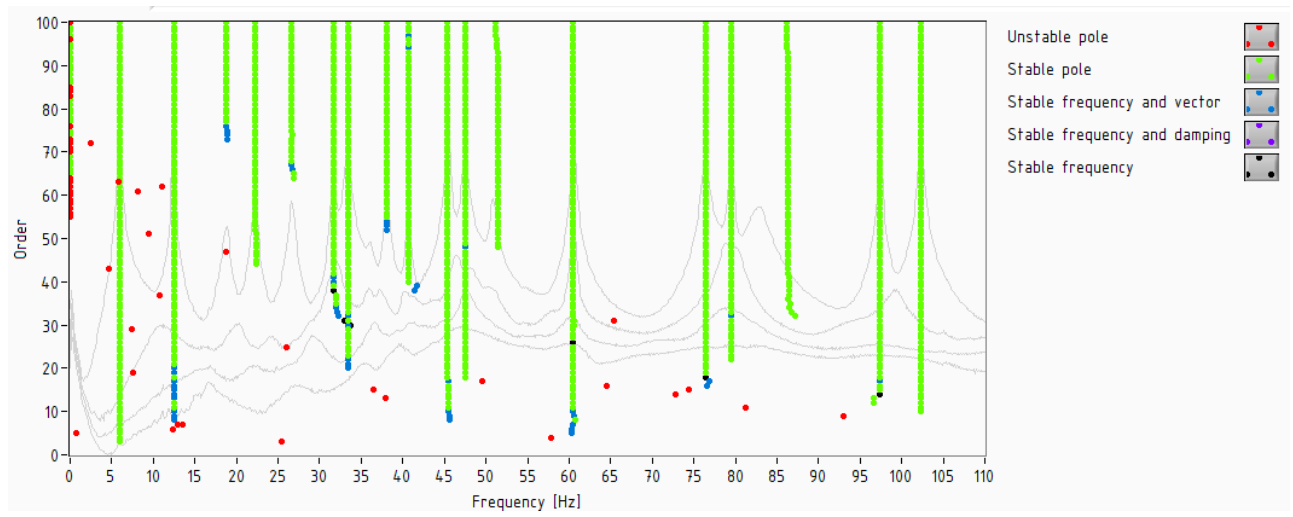

Fig. 3. The result of SSI method. 
A stabilization diagram is used to determine the natural frequencies for SSI-COV method, Fig. 3. Values are calculated for the maximum model order of 100. All modes were determined for model order of 80. A natural frequencies comparison of both methods can be seen in the following table and mode shapes from FDD method are illustrated in Fig. 4.

Table 1. Comparison of Frequency Domain Decomposition method and Stochastic Subspace.

\begin{tabular}{|c|c|c|c|c|c|c|c|c|}
\hline & 1 & 2 & 3 & 4 & 5 & 6 & 7 & 8 \\
\hline \hline FDD & 5.87 & 12.37 & 22.12 & 26.50 & 31.62 & 33.37 & 38.00 & 40.62 \\
\hline SSI (model order 80) & 5.92 & 12.44 & 22.29 & 26.66 & 31.66 & 33.38 & 38.10 & 40.68 \\
\hline difference [\%] & 0.84 & 0.56 & 0.76 & 0.60 & 0.13 & 0.03 & 0.26 & 0.15 \\
\hline
\end{tabular}

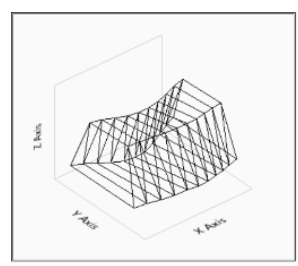

1.freq $5.87 \mathrm{~Hz}$

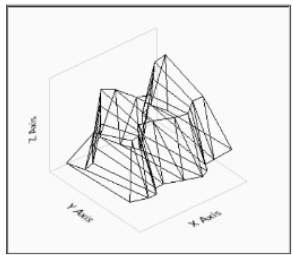

5.freq $31.62 \mathrm{~Hz}$

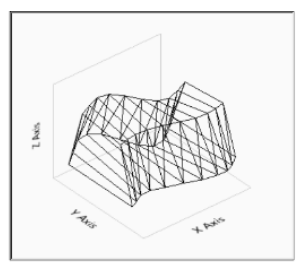

2.freq $12.37 \mathrm{~Hz}$

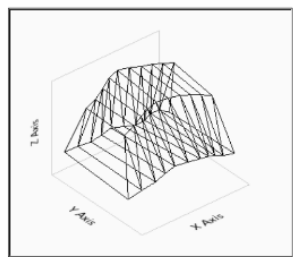

6.freq $33.37 \mathrm{~Hz}$

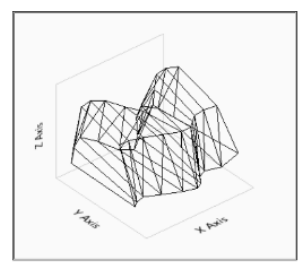

3.freq $22.12 \mathrm{~Hz}$

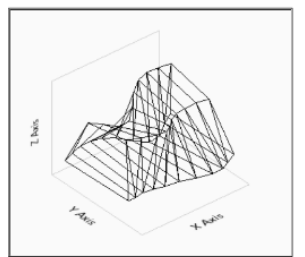

7.freq $38.00 \mathrm{~Hz}$

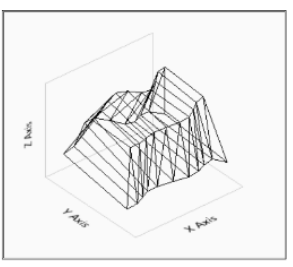

4.freq $26.50 \mathrm{~Hz}$

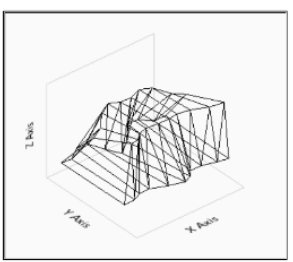

8.freq $40.62 \mathrm{~Hz}$

Fig. 4. Mode shapes obtained from FDD algorithm.

It is clear from the calculated shapes that the truss structure tends to oscillate in bending and torsion shapes due to its shape. This is because the transverse stiffness for both the horizontal and vertical directions is very similar.

\section{Conclusions}

A comparison of the resulting natural frequencies in Table 1 shows that similar results are obtained for both methods and the frequency difference is less than $1 \%$. In the case of the SSI method, it is important to choose a higher model order than 50 to reach correct results. All estimated natural frequencies by SSI-COV method are higher than those estimated by FDD. The advantage of SSI method is that it requires less user interaction in the analysis task. However, in some cases this could be its disadvantage as the modes not representing the structural response of the structure may be identified. Therefore, the combination of methods seems to be a suitable. 
This work was supported under the project of Operational Programme Research and Innovation: Research and development activities of the University of Zilina in the Industry of 21 st century in the field of materials and nanotechnologies, No. 313011T426. The project is co-funding by the European Regional Development Fund and VEGA 1/0006/20 Constructions of transport structures in terms of dynamic analysis of real operational states.

\section{References}

1. J. J. Sinou, A review of damage detection and health monitoring of mechanical systems from changes in the measurement of linear and non-linear vibrations (2009)

2. Nuno M.M. Maia, Júlio M.M. Silva, Theoretical and Experimental Modal Analysis (Research Studies Press LTD, July, 1998)

3. M. Venglár, M. Sokol, R. Ároch, Ambient vibration measurements of steel truss bridges. Journal of Measurements in Engineering 6, 4, 234-239 (2018)

4. R. Brincker, L. Zhang, P. Andersen, Modal identification of output-only systems using frequency domain decomposition. Smart materials and structures, 10, 3, 441 (2001)

5. I. G. Araújo, J. E. Laier, Operational modal analysis using SVD of power spectral density transmissibility matrices. Mechanical Systems and Signal Processing, 46, 1, 129-145 (2014)

6. M. Döhler, L. Mevel, Modular subspace-based system identification from multi-setup measurements. IEEE Transactions on Automatic Control, 57, 11, 2951-2956 (2012)

7. B. Peeters, System identification and damage detection in civil engineering (2000)

8. M. Döhler, Subspace-based system identification and fault detection: Algorithms for large systems and application to structural vibration analysis, PhD Thesis, (2011)

9. J. Kortiš, et al. Experimental modal test of the laboratory model of steel truss structure. Civil and Environmental Engineering, 12, 2, 116-121 (2016)

10. E. Parloo, P. Guillaume, B. Cauberghe, Maximum likelihood identification of nonstationary operational data. Journal of Sound and Vibration, 268 , 5, 971-991 (2003) 